\title{
Development of High Signal Intensity within the Globus Pallidus and Dentate Nucleus following Multiple Administrations of Gadobenate Dimeglumine
}

\author{
(1D G.M. Bolles, DM. Yazdani, DS.T. Stalcup, (D) S.G. Creeden, (DH.R. Collins, DP.J. Nietert, and DD.R. Roberts
}

\begin{abstract}
BACKGROUND AND PURPOSE: Previous studies have evaluated various gadolinium based contrast agents and their association with gadolinium retention, however, there is a discrepancy in the literature concerning the linear agent gadobenate dimeglumine. Our aim was to determine whether an association exists between the administration of gadobenate dimeglumine and the development of intrinsic T7-weighted signal in the dentate nucleus and globus pallidus.
\end{abstract}

MATERIALS AND METHODS: In this single-center, retrospective study, the signal intensity of the globus pallidus, dentate nucleus, thalamus, and middle cerebellar peduncle was measured on unenhanced T1-weighted images in 29 adult patients who had undergone multiple contrast MRIs using exclusively gadobenate dimeglumine (mean, $10.1 \pm 3.23$ doses; range, 6-18 doses). Two neuroradiologists, blinded to the number of prior gadolinium-based contrast agent administrations, separately placed ROls within the globi pallidi, thalami, dentate nuclei, and middle cerebellar peduncles on the last MR imaging examinations. The correlations between the globus pallidus:thalamus and the dentate nucleus:middle cerebellar peduncle signal intensity ratios with the number of gadolinium-based contrast agent administrations and cumulative dose were tested with either 1-tailed Pearson or Spearman correlations. A priori, $P<.05$ was considered statistically significant.

RESULTS: Both the globus pallidus:thalamus and dentate nucleus:middle cerebellar peduncle ratios showed significant correlation with the number of gadolinium-based contrast agent administrations $(r=0.39, P=.017$, and $r=0.58, P=.001$, respectively). Additionally, the globus pallidus:thalamus and dentate nucleus:middle cerebellar peduncle ratios showed significant correlation with the cumulative dose of gadobenate dimeglumine $(r=0.48, P=.004$, and $r=0.43, P=.009$, respectively). Dentate nucleus hyperintensity was qualitatively present on the last MR imaging in $79.3 \%-86.2 \%$ of patients and in all patients who had received $>10$ doses.

CONCLUSIONS: At high cumulative doses (commonly experienced by patients, for example, with neoplastic disease), gadobenate dimeglumine is associated with an increase in the globus pallidus:thalamus and dentate nucleus:middle cerebellar peduncles signal intensity ratios.

ABBREVIATIONS: $\mathrm{DN}=$ dentate nucleus; $\mathrm{GBCA}=$ gadolinium-based contrast agent; $\mathrm{GP}=$ globus pallidus; $\mathrm{MCP}=$ middle cerebellar peduncle; $\mathrm{TH}=$ thalamus

G adolinium-based contrast agents (GBCAs) are extensively used in evaluating both central nervous system and nonCNS pathologies with MR imaging and provide critical diagnostic

Received August 29, 2017; accepted after revision October 30.

From the Department of Radiology and Radiological Sciences (G.M.B., M.Y., S.T.S., S.G.C., H.R.C., D.R.R.), Department of Neuroradiology, and Department of Public Health Sciences (P.J.N.), Medical University of South Carolina, Charleston, South Carolina.

Paper previously presented at: Annual Meeting of the American Society of Neuroradiology and the Foundation of the ASNR Symposium, April 22-27, 2017; Long Beach, California.

Dr Nietert's time on this project was funded, in part, by the National Institutes of Health National Center for Advancing Translational Science (grant No. UL1TR001450) and the National Institute of General Medical Sciences (grant No. U54-GM104941)

Please address correspondence to Donna R. Roberts, MD, Department of Radiology and Radiological Sciences, 6 Jonathan Lucas St, MSC 323, Medical University of South Carolina, Charleston, SC 29425-3230; e-mail: robertdr@musc.edu and treatment-guiding information. All GBCAs are composed of gadolinium and a chelating agent. Because gadolinium is an extremely toxic substance, chelating agents are designed to prevent release of gadolinium in the body before renal excretion.

In 2014, Kanda et $\mathrm{al}^{1}$ first reported the development of progressively increasing signal intensity in the globus pallidus (GP) and dentate nucleus (DN) on unenhanced T1-weighted imaging in patients who had undergone multiple contrast MRIs with the administration of gadopentetate dimeglumine or gadodiamide. At postmortem examination, the cause of this high T1-weighted signal intensity in the GP and DN was shown to be retained gadolinium. $^{2,3}$

Subsequently, several studies evaluated various GBCAs and

\footnotetext{
- Indicates open access to non-subscribers at www.ajnr.org

http://dx.doi.org/10.3174/ajnr.A5510
} 
their association with gadolinium retention in both the adult ${ }^{4-11}$ and pediatric populations. ${ }^{12-19}$ The general consensus is that linear, but not macrocyclic, GBCAs are associated with the development of hyperintense signal in the GP and DN on unenhanced T1-weighted images as demonstrated by most of these studies, though controversial findings remain. ${ }^{20-23}$ There is, however, discrepancy in the literature concerning the linear agent gadobenate dimeglumine. ${ }^{24-26}$ Ramalho et $\mathrm{al}^{24}$ evaluated the signal intensity of the DN and GP in patients who received either gadodiamide, a linear nonionic GBCA, or gadobenate dimeglumine, a linear ionic contrast agent. While the administration of multiple doses of gadodiamide was associated with a significant increase in DN and GP T1-weighted signal intensity, the authors found only a significant trend toward an increase in relative percentage change in signal intensity for the DN in patients receiving multiple doses of gadobenate dimeglumine. However, the patients in this study received a mean of $4.6 \pm 2.2$ doses (range, 3-11 doses), whereas most studies have found the development of high T1-weighted signal in the DN only after $>5$ GBCA administrations. ${ }^{27}$

Likewise, Schneider et $\mathrm{al}^{26}$ reported no increase in the T1weighted signal intensity of the DN in pediatric patients who had been exposed to a mean of 7.8 injections of gadobenate dimeglumine; however, the agent was administered at half dose $(0.05$ $\mathrm{mmol} / \mathrm{kg}$ ). On the other hand, a study by Weberling et al, ${ }^{25}$ found a significant increase in the T1-weighted signal intensity in the DN after serial injections of gadobenate dimeglumine but not a significant correlation between the DN-to-pons ratio and the accumulated dose. The patient population in this study was predominantly those with melanoma (47/50 patients), and 14 patients had undergone radiation therapy, which may have confounded the results.

Given the discrepancies in the literature, the purpose of this study was to provide further evaluation of gadobenate dimeglumine by assessing a group of patients with various pathologies and no prior radiation exposure to the posterior fossa who had received at least 7 cumulative injections of exclusively gadobenate dimeglumine administered at the full FDA-approved dose of 0.1 $\mathrm{mmol} / \mathrm{kg}$.

\section{MATERIALS AND METHODS}

This single-center, retrospective, cohort study was performed after institutional review board approval, with a waiver of informed consent, in compliance with the Health Insurance Portability and Accountability Act. The biostatistician's time was covered by several National Institutes of Health grants. Otherwise, the study was unsponsored.

\section{Patients}

All patients at our institution who had undergone MR imaging of the brain with contrast administration from January 2013 until July 2016 were screened for inclusion in the study with the following criteria: 1) adult patients 18 years of age and older, 2) a minimum of 7 gadolinium-enhanced MR imaging scans (which included the last MR imaging for reference), and 3) all contrast MR imaging examinations performed exclusively with gadobenate dimeglumine. During this time, 11,669 MRIs of the brain with and without gadobenate dimeglumine were performed, and 222 patients had received at least 7 doses of gadobenate dimeglumine.

Using both the local PACS and the electronic medical record, we excluded all patients who had undergone a contrast MR imaging outside our facility at any time to ensure documentation of all GBCA administrations. Additionally, to avoid possible confounding variables, we excluded patients with the following: 1) posterior fossa disease obscuring the dentate nucleus or middle cerebellar peduncle, 2) radiation exposure to the posterior fossa, 3) multiple sclerosis or other disease processes associated with intracranial intrinsic T1-shortening, 4) unsatisfactory images due to MR imaging or motion artifacts affecting the evaluation of the posterior fossa, and 5) any history of abnormal renal (estimated glomerular filtration rate at the time of imaging of $<30 \mathrm{~mL} / \mathrm{min}$ per $1.73 \mathrm{~m}^{2}$ ) or hepatic function as evaluated by routine laboratory tests.

\section{Imaging Protocols}

MR imaging was performed on either a 3T scanner (Skyra and/or Verio; Siemens, Erlangen, Germany) or a $1.5 \mathrm{~T}$ scanner (Aera and/or Avanto; Siemens). The standard 1.5T MR imaging protocol included either axial T1-weighted spin-echo (TR, 500-588 $\mathrm{ms}$; TE, 7.7-12 ms; slice thickness, 5-mm; gap 1.0) or 3D MPRAGE (TR, 1780-2200 ms; TE, 2.91-3.37 ms; slice thickness, $1.0 \mathrm{~mm}$; gap, 0.5; FOV, $250 \mathrm{~mm}$ ) before and after GBCA administration. The standard 3T MR imaging protocol included either axial T1-weighted spin-echo (TR, 500-550 ms; TE, 6.4$8.9 \mathrm{~ms}$; slice thickness, $5 \mathrm{~mm}$; gap, 1.0) or 3D MPRAGE (TR, 1690-2300 ms; TE, 2.3-3.2 ms; slice thickness, $1.0 \mathrm{~mm}$; gap, 0.5 ; FOV, $250 \mathrm{~mm}$ ) before and after GBCA administration. Gadobenate dimeglumine was administered as an intravenous bolus using body weight-adjusted dosing at $0.1 \mathrm{mmol} / \mathrm{kg}$ per departmental protocol.

\section{Image and Data Analysis}

Two neuroradiologists (M.Y. and S.T.S.), with 3 and 4 years of experience respectively, evaluated the images. Each reader was independently presented with the initial and final MR imaging of each patient in a randomized, blinded fashion on the PACS workstation and asked to qualitatively determine whether the DN demonstrated T1-weighted hyperintensity on the unenhanced T1-weighted images. The readers were blinded to the number of prior GBCA administrations.

In addition, each reader was asked to place ROIs by visual analysis within the GP, thalamus (TH), DN, and middle cerebellar peduncle (MCP) bilaterally, as described by Kanda et al, ${ }^{1}$ on the last MR imaging scan. The mean signal intensity of each ROI was obtained and used to calculate the DN-to-MCP signal intensity ratio (DN:MCP) and the GP-to-TH signal intensity ratio (GP: $\mathrm{TH}$ ) for each subject.

\section{Statistical Analysis}

To test the concordance between the 2 readers concerning the GP, $\mathrm{TH}, \mathrm{DN}$, and MCP mean signal intensity measurements, we calculated the Lin concordance correlation coefficient using on-line software (https://www.niwa.co.nz/node/104318/concordance).

The Shapiro-Wilk test was used to assess the normality of the data. For the GP:TH ratios, the $P$ value was $=.005$, indicating that 
Total No. of patients

Age (yr)

Sex (male/female)

No. of enhanced MRI scans ${ }^{b}$

Cumulative dose $(\mathrm{mL})^{\mathrm{b}}$

Interval (days) between 1st and

final MRI

Diagnoses

\section{9} $20: 9$

$10.1 \pm 3.2(6-18)$

$175.2 \pm 59.1(76-316)$

$658.5 \pm 250.5(154-1154)$

Intracranial metastases $(n=10)$, primary brain neoplasm $(n=11)$, meningioma $(n=3)$, melanomas $(n=3)$, unknown lesion $(n=2)$
$52.1 \pm 17.1(18-84)$

\footnotetext{
${ }^{a}$ Data are mean $\pm S D$, with the range in parentheses.
}

${ }^{\mathrm{b}}$ Does not include the last MRI used for measurement.

the data were not normally distributed; therefore, a Spearman correlation was used to investigate the relationship between the GP:TH ratios on the final MR imaging examination and the number of administered GBCA doses as well as the total cumulative dose. For the DN:MCP ratios, the $P$ value was 0.786 , indicating a normal distribution; therefore, a Pearson correlation was used to assess the relationship between the DN:MCP ratios on the final MR imaging examination and the number of administered GBCA doses and the total cumulative dose. Statistical significance was considered at the $\alpha=.05$ threshold, and 1-tailed $P$ values are reported (SPSS, Version 23; IBM, Armonk, New York).

Interrater reliability concerning the presence or absence of hyperintense T1-weighted signal within the dentate nucleus was assessed using the Cohen $\kappa$ to determine the level of agreement between the 2 readers with the magnitude interpreted using common guidelines. ${ }^{28,29}$

\section{RESULTS}

Twenty-nine patients met the inclusion/exclusion criteria during the study period, and the patient characteristics are described in the Table. The mean age of the patients was $52.1 \pm 17.1$ years. There were 20 men and 9 women. Most patients underwent imaging for the diagnosis of neoplasm. The mean number of prior GBCA administrations was $10.1 \pm 3.23$ doses (range, $6-18$ doses). Note that 2 patients received only 7 GBCA administrations; therefore, at the time of the last enhanced MR imaging used for measurement, these 2 patients had previously received 6 GBCA doses. Cumulative doses of gadobenate dimeglumine ranged from 76 to $316 \mathrm{~mL}$, with a mean of $175.2 \pm 59.05 \mathrm{~mL}$.

Concerning ROI measurements within the GP, TH, DN, and MCP, the value of the Lin correlation coefficient between readers ranged from 0.97 to 0.99 , indicating substantial-to-near-perfect reader concordance. Therefore, the measurements from both readers were averaged.

The GP:TH and DN:MCP ratios on the last MR imaging both showed a significant correlation with the number of previous GBCA administrations: $r=0.39, P=.017$, and $r=0.58, P=.001$, respectively (Fig 1 ). There was also a significant correlation between the cumulative doses of gadobenate dimeglumine and the last GP:TH ratios $(r=0.48, P=.004)$ and the last DN:MCP ratios $(r=0.43, P=.009)$.

Higher doses of gadobenate dimeglumine resulted in qualitatively visible hyperintensity of the dentate nucleus on the final MR imaging examination as reported by readers 1 and 2 in $79.3 \%$ and $86.2 \%$ of the patients, respectively (Fig $2)$. Both readers reported dentate nucleus hyperintensity in all patients who had received $>10$ doses of gadobenate dimeglumine. Concerning agreement between the 2 readers on the qualitative presence or absence of T1-weighted hyperintensity within the dentate nucleus on the final MR imaging, $\kappa=0.77$, $P<.0005$ indicated substantial interobserver agreement.

\section{DISCUSSION}

In this study, we demonstrate significant positive correlations between the GP:TH and the DN:MCP ratios and the number of previous GBCA administrations and the total cumulative dose. Our findings are in agreement with the study of Weberling et $\mathrm{al}^{25}$ and extend those results to patients with various intracranial pathologies without the potential confounding effect of radiation therapy.

In our study, hyperintensity within the GP and DN was demonstrated following the administration of a mean of $10.1 \pm 3.2$ doses. On the other hand, no significant increase in $\mathrm{T} 1$ signal on unenhanced images was seen in the GP or DN in the study of Ramalho et al, ${ }^{24}$ following the administration of a mean of $4.6 \pm$ 2.2 doses of gadobenate dimeglumine, but it was present following gadodiamide administered at a mean of $5.0 \pm 2.4$ doses. The larger mean dose of gadobenate dimeglumine in our study likely explains this discrepancy. Whether the mean dose required to produce T1 shortening on MR imaging is reflective of the rate or the amount of gadolinium deposition associated with a specific agent is unclear because several confounding factors influence the appearance of gadolinium on MR imaging, including the specific molecular species present. Animal and human postmortem studies will be helpful in comparing the various agents and quantifying their propensity for gadolinium deposition.

As a GBCA, gadobenate dimeglumine has several unique properties. Due to weak, transient interactions with serum albumin, ${ }^{30,31}$ gadobenate dimeglumine has high $\mathrm{R} 1$ relaxivity in vivo, ${ }^{32,33}$ resulting in greater signal intensity enhancement on T1weighted images. In addition to renal excretion, a small percentage of gadobenate dimeglumine is excreted by the hepatobiliary system. ${ }^{34}$ While gadobenate dimeglumine is a linear GBCA, it has not been associated with a high risk for nephrogenic systemic fibrosis. ${ }^{35-37}$ However, in animal ${ }^{38,39}$ and human serum ${ }^{40}$ studies, gadobenate dimeglumine behaves as would be expected for a linear agent. Therefore, considering the unique properties of gadobenate dimeglumine, it is important to determine whether the agent is associated with T1-hyperintensities within specific regions of the brain. This knowledge is important for performing a clinical risk-versus-benefit analysis.

There were several limitations to this study. Patients could have received GBCA administrations at an outside institution without reporting this information to their clinicians; however, in addition to searching the PACS system for imported outside imaging, the medical records were thoroughly screened for any doc- 

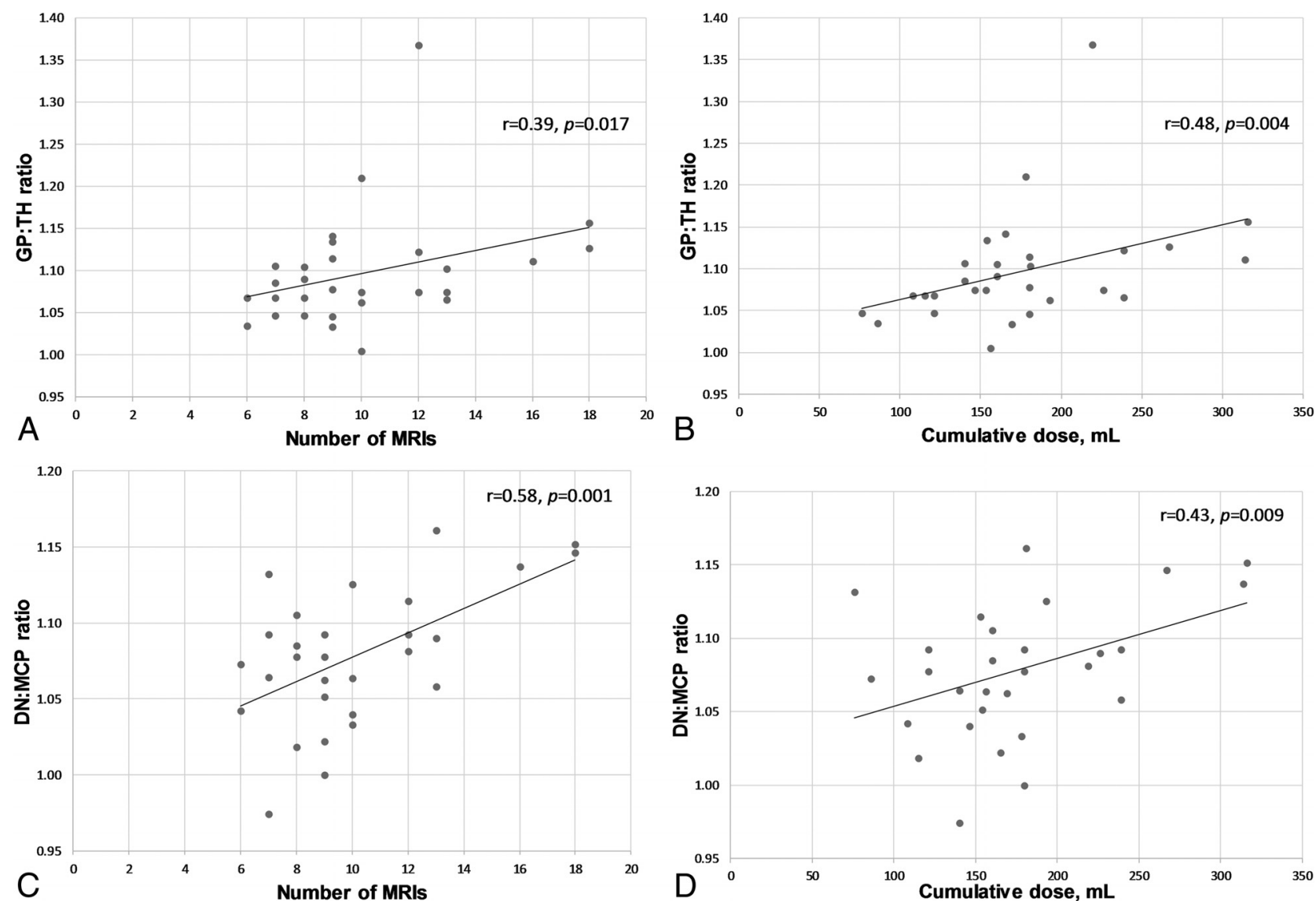

FIG 1. Graphs of the GP:TH and DN:MCP ratios plotted against the number of GBCA enhanced MR imaging scans $(A$ and $C)$ and the cumulative dose of gadobenate dimeglumine $(B$ and $D)$.

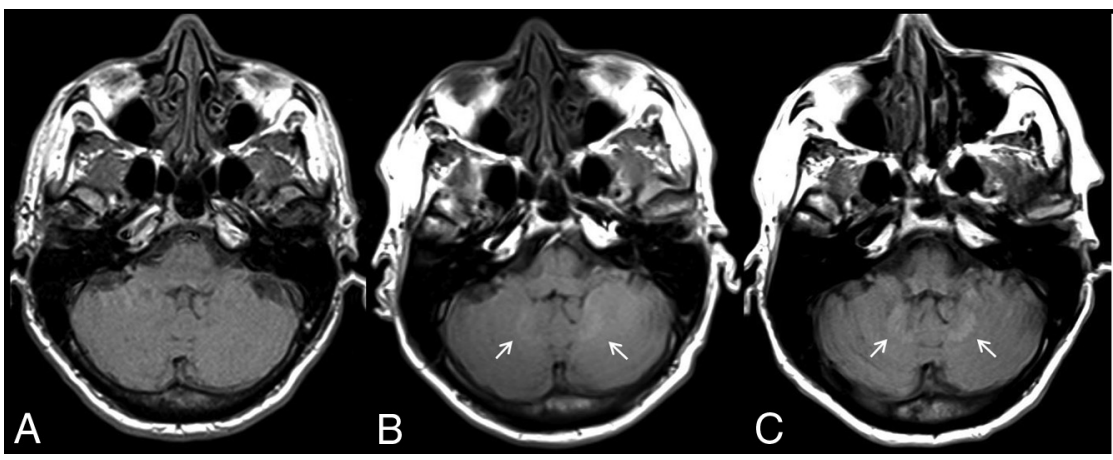

FIG 2. Unenhanced, axial T7-weighted MR images through the dentate nucleus in a 54-year-old man with metastatic renal cell carcinoma after the administration of $1(A), 8(B)$, and $18(C)$ doses of gadobenate dimeglumine. There is a progressive increase in the $\mathrm{T} 1$ signal intensity in the dentate nucleus (arrows).

umentation of imaging before presentation to our institution. Varying field strengths and MR imaging parameters were used and precluded the calculation of relative percentage change. Finally, as in all studies of this nature, ROI placement is somewhat subjective and therefore subject to reader bias; however, the readers were blinded to the number of prior GBCA administrations; thus, this subjectivity is not likely to have confounded our study findings.

As mentioned in prior articles on the same topic, further studies, particularly those using tissue analysis, should continue to evaluate specific GBCAs and their propensity to deposit within the human body. Furthermore, research evaluating any potential clinical sequelae from gadolinium deposition, both within the brain and other body tissues, should be performed. Continued prudence in selecting patients who should receive contrast-enhanced MRIs is recommended.

\section{CONCLUSIONS}

At higher cumulative doses, as can be experienced by patient groups, such as those with tumors, gadobenate dimeglumine is associated with a significant increase in the GP:TH and DN:MCP ratios on unenhanced T1-weighted images. These data should be incorporated into a risk-versus-benefit analysis when determining the need for GBCA administration in individual patients. While any potential clinical significance is unknown, the iatrogenic deposition of gadolinium within sensitive tissues such as the brain should be minimized in situations where alternative agents with similar clinical efficacy are available.

Disclosures: Paul J. Nietert—RELATED: Grant: National Institutes of Health.* Donna R. Roberts-UNRELATED: Consultancy: Guerbet, Comments: advisory board member; Grants/Grants Pending: Guerbet, Comments: investigator-initiated award site Principal Investigator, clinical trial*; Travel/Accommodations/Meeting Expenses Unrelated to Activities Listed: Guerbet. *Money paid to the institution. 


\section{REFERENCES}

1. Kanda $\mathrm{T}$, Ishii $\mathrm{K}$, Kawaguchi $\mathrm{H}$, et al. High signal intensity in the dentate nucleus and globus pallidus on unenhanced T1weighted MR images: relationship with increasing cumulative dose of a gadolinium-based contrast material. Radiology 2014; 270:834-41 CrossRef Medline

2. Kanda T, Fukusato T, Matsuda M, et al. Gadolinium-based contrast agent accumulates in the brain even in subjects without severe renal dysfunction: evaluation of autopsy brain specimens with inductively coupled plasma mass spectroscopy. Radiology 2015;276: 228-32 CrossRef Medline

3. McDonald RJ, McDonald JS, Kallmes DF, et al. Intracranial gadolinium deposition after contrast-enhanced MR imaging. Radiology 2015;275:772-82 CrossRef Medline

4. Errante Y, Cirimele V, Mallio CA, et al. Progressive increase of T1 signal intensity of the dentate nucleus on unenhanced magnetic resonance images is associated with cumulative doses of intravenously administered gadodiamide in patients with normal renal function, suggesting dechelation. Invest Radiol 2014;49:685-90 CrossRef Medline

5. Quattrocchi CC, Mallio CA, Errante Y, et al. Gadodiamide and dentate nucleus $\mathrm{T} 1$ hyperintensity in patients with meningioma evaluated by multiple follow-up contrast-enhanced magnetic resonance examinations with no systemic interval therapy. Invest Radiol 2015; 50:470-72 CrossRef Medline

6. Radbruch A, Weberling LD, Kieslich PJ, et al. High-signal intensity in the dentate nucleus and globus pallidus on unenhanced T1weighted images: evaluation of the macrocyclic gadolinium-based contrast agent gadobutrol. Invest Radiol 2015;50:805-10 CrossRef Medline

7. Cao Y, Huang DQ, Shih G, et al. Signal change in the dentate nucleus on T1-weighted MR images after multiple administrations of gadopentetate dimeglumine versus gadobutrol. AJR Am J Roentgenol 2016;206:414-19 CrossRef Medline

8. Stojanov DA, Aracki-Trenkic A, Vojinovic S, et al. Increasing signal intensity within the dentate nucleus and globus pallidus on unenhanced T1W magnetic resonance images in patients with relapsingremitting multiple sclerosis: correlation with cumulative dose of a macrocyclic gadolinium-based contrast agent, gadobutrol. Eur Radiol 2016;26:807-15 CrossRef Medline

9. Bjørnerud A, Vatnehol SA, Larsson C, et al. Signal enhancement of the dentate nucleus at unenhanced MR imaging after very high cumulative doses of the macrocyclic gadolinium-based contrast agent gadobutrol: an observational study. Radiology 2017;285:434-44 CrossRef Medline

10. Radbruch A, Haase R, Kieslich PJ, et al. No signal intensity increase in the dentate nucleus on unenhanced T1-weighted MR images after more than 20 serial injections of macrocyclic gadolinium-based contrast agents. Radiology 2017;282:699-707 CrossRef Medline

11. Splendiani A, Perri M, Marsecano C, et al. Effects of serial macrocyclic-based contrast materials gadoterate meglumine and gadobutrol administrations on gadolinium-related dentate nuclei signal increases in unenhanced T1-weighted brain: a retrospective study in $\mathbf{1 5 8}$ multiple sclerosis (MS) patients. Radiol Med $2017 \mathrm{Sep}$ 27. [Epub ahead of print] CrossRef Medline

12. Miller JH, Hu HH, Pokorney A, et al. MRI brain signal intensity changes of a child during the course of 35 gadolinium contrast examinations. Pediatrics 2015;136:e1637-40 CrossRef Medline

13. Roberts DR, Chatterjee AR, Yazdani M, et al. Pediatric patients demonstrate progressive T1-weighted hyperintensity in the dentate nucleus following multiple doses of gadolinium-based contrast agent. AJNR Am J Neuroradiol 2016;37:2340 -47 CrossRef Medline

14. Roberts DR, Holden KR. Progressive increase of T1 signal intensity in the dentate nucleus and globus pallidus on unenhanced T1weighted MR images in the pediatric brain exposed to multiple doses of gadolinium contrast. Brain Dev 2016;38:331-36 CrossRef Medline

15. Hu HH, Pokorney A, Towbin RB, et al. Increased signal intensi- ties in the dentate nucleus and globus pallidus on unenhanced T1-weighted images: evidence in children undergoing multiple gadolinium MRI exams. Pediatr Radiol 2016;46:1590-98 CrossRef Medline

16. Flood TF, Stence NV, Maloney JA, et al. Pediatric brain: repeated exposure to linear gadolinium-based contrast material is associated with increased signal intensity at unenhanced T1-weighted MR imaging. Radiology 2017;282:222-28 CrossRef Medline

17. Radbruch A, Haase R, Kickingereder $P$, et al. Pediatric brain: no increased signal intensity in the dentate nucleus on unenhanced T1-weighted MR images after consecutive exposure to a macrocyclic gadolinium-based contrast agent. Radiology 2017;283:828-36 CrossRef Medline

18. Tibussek D, Rademacher C, Caspers J, et al. Gadolinium brain deposition after macrocyclic gadolinium administration: a pediatric case-control study. Radiology 2017;285:223-230 CrossRef Medline

19. Rossi Espagnet MC, Bernardi B, Pasquini L, et al. Signal intensity at unenhanced T1-weighted magnetic resonance in the globus pallidus and dentate nucleus after serial administrations of a macrocyclic gadolinium-based contrast agent in children. Pediatr Radiol 2017;47:1345-52 CrossRef Medline

20. Kanda $\mathrm{T}$, Osawa $\mathrm{M}$, Oba $\mathrm{H}$, et al. High signal intensity in dentate nucleus on unenhanced T1-weighted MR images: association with linear versus macrocyclic gadolinium chelate administration. $R a$ diology 2015;275:803-09 CrossRef Medline

21. Radbruch A, Quattrocchi CC. Interpreting signal-intensity ratios without visible $\mathrm{T} 1$ hyperintensities in clinical gadolinium retention studies. Pediatr Radiol 2017;47:1688-89 CrossRef Medline

22. Radbruch A, Weberling LD, Kieslich PJ, et al. Gadolinium retention in the dentate nucleus and globus pallidus is dependent on the class of contrast agent. Radiology 2015;275:783-91 CrossRef Medline

23. Radbruch A, Weberling LD, Kieslich PJ, et al. Intraindividual analysis of signal intensity changes in the dentate nucleus after consecutive serial applications of linear and macrocyclic gadolinium-based contrast agents. Invest Radiol 2016;51:683-90 CrossRef Medline

24. Ramalho J, Castillo M, AlObaidy M, et al. High signal intensity in globus pallidus and dentate nucleus on unenhanced T1-weighted MR images: evaluation of two linear gadolinium-based contrast agents. Radiology 2015;276:836-44 CrossRef Medline

25. Weberling LD, Kieslich PJ, Kickingereder P, et al. Increased signal intensity in the dentate nucleus on unenhanced T1-weighted images after gadobenate dimeglumine administration. Invest Radiol 2015;50:743-48 CrossRef Medline

26. Schneider GK, Stroeder J, Roditi G, et al. T1 signal measurements in pediatric brain: findings after multiple exposures to gadobenate dimeglumine for imaging of nonneurologic disease. AJNR Am J Neuroradiol 2017;38:1799-1806 CrossRef Medline

27. Kanda $\mathrm{T}$, Oba $\mathrm{H}$, Toyoda $\mathrm{K}$, et al. Brain gadolinium deposition after administration of gadolinium-based contrast agents. Jpn J Radiol 2016;34:3-9 CrossRef Medline

28. Cohen J. Weighted kappa: nominal scale agreement with provision for scaled disagreement or partial credit. Psychol Bull 1968;70: 213-20 CrossRef Medline

29. Landis JR, Koch GG. The measurement of observer agreement for categorical data. Biometrics 1977;33:159-74 CrossRef Medline

30. Cavagna FM, Maggioni F, Castelli PM, et al. Gadolinium chelates with weak binding to serum proteins: a new class of high-efficiency, general purpose contrast agents for magnetic resonance imaging. Invest Radiol 1997;32:780-96 CrossRef Medline

31. de Haën C, Cabrini M, Akhnana L, et al. Gadobenate dimeglumine $0.5 \mathrm{M}$ solution for injection (MultiHance) pharmaceutical formulation and physicochemical properties of a new magnetic resonance imaging contrast medium. J Comput Assist Tomogr 1999;23(Suppl 1):S161-68 CrossRef Medline

32. Pintaske J, Martirosian P, Graf $\mathrm{H}$, et al. Relaxivity of gadopentetate dimeglumine (Magnevist), gadobutrol (Gadovist), and gadobenate dimeglumine (MultiHance) in human blood plasma at 0.2, 1.5, and 3 Tesla. Invest Radiol 2006;41:213-21 CrossRef Medline 
33. Shen Y, Goerner FL, Snyder C, et al. T1 relaxivities of gadoliniumbased magnetic resonance contrast agents in human whole blood at 1.5, 3, and 7 T. Invest Radiol 2015;50:330-38 CrossRef Medline

34. Kirchin MA, Pirovano GP, Spinazzi A. Gadobenate dimeglumine (GdBOPTA): an overview. Invest Radiol 1998;33:798-809 CrossRef Medline

35. Bruce R, Wentland AL, Haemel AK, et al. Incidence of nephrogenic systemic fibrosis using gadobenate dimeglumine in 1423 patients with renal insufficiency compared with gadodiamide. Invest Radiol 2016;51:701-05 CrossRef Medline

36. Nandwana SB, Moreno CC, Osipow MT, et al. Gadobenate dimeglumine administration and nephrogenic systemic fibrosis: is there a real risk in patients with impaired renal function? Radiology 2015; 276:741-47 CrossRef Medline

37. Martin DR, Kalb B, Mittal A, et al. No Incidence of nephrogenic systemic fibrosis after gadobenate dimeglumine administration in pa- tients undergoing dialysis or those with severe chronic kidney disease. Radiology 2017 Jul 21. [Epub ahead of print] CrossRef Medline

38. Frenzel T, Apte C, Jost G, et al. Quantification and assessment of the chemical form of residual gadolinium in the brain after repeated administration of gadolinium-based contrast agents: comparative study in rats. Invest Radiol 2017;52:396-404 CrossRef Medline

39. McDonald RJ, McDonald JS, Dai D, et al. Comparison of gadolinium concentrations within multiple rat organs after intravenous administration of linear versus macrocyclic gadolinium chelates. $R a$ diology 2017;285:536-45 CrossRef Medline

40. Frenzel T, Lengsfeld P, Schirmer H, et al. Stability of gadoliniumbased magnetic resonance imaging contrast agents in human serum at 37 degrees C. Invest Radiol 2008;43:817-28 CrossRef Medline 